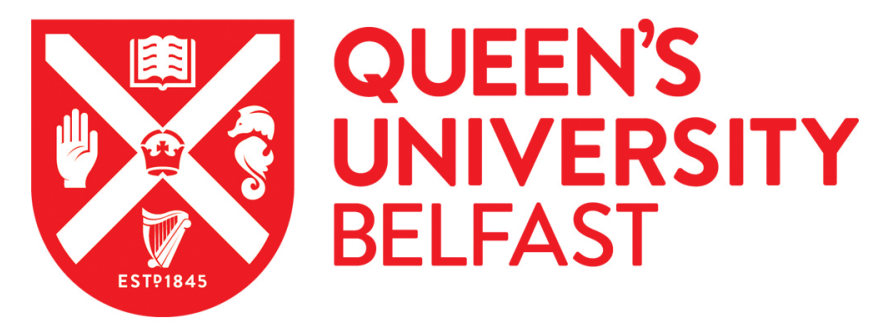

\title{
Dense spectrum of resonances and spin-1/2 particle capture in a near-black-hole metric
}

Gossel, G. H., Berengut, J. C., Flambaum, V. V., \& Gribakin, G. F. (2013). Dense spectrum of resonances and spin-1/2 particle capture in a near-black-hole metric. Physical Review D - Particles, Fields, Gravitation and Cosmology, 88(2), [027501]. https://doi.org/10.1103/PhysRevD.88.027501

Published in:

Physical Review D - Particles, Fields, Gravitation and Cosmology

Document Version:

Peer reviewed version

Queen's University Belfast - Research Portal:

Link to publication record in Queen's University Belfast Research Portal

Publisher rights

(C) 2013 American Physical Society

\section{General rights}

Copyright for the publications made accessible via the Queen's University Belfast Research Portal is retained by the author(s) and / or other copyright owners and it is a condition of accessing these publications that users recognise and abide by the legal requirements associated with these rights.

Take down policy

The Research Portal is Queen's institutional repository that provides access to Queen's research output. Every effort has been made to ensure that content in the Research Portal does not infringe any person's rights, or applicable UK laws. If you discover content in the Research Portal that you believe breaches copyright or violates any law, please contact openaccess@qub.ac.uk. 


\title{
Dense spectrum of resonances and spin- $1 / 2$ particle capture in a near-black-hole metric
}

G. H. Gossel, J. C. Berengut, and V. V. Flambaum

School of Physics, University of New South Wales, Sydney 2052, Australia

\author{
G. F. Gribakin \\ School of Mathematics and Physics, Queen's University, Belfast BT7 1NN, Northern Ireland, UK
}

(Dated: June 23, 2013)

\begin{abstract}
We show that a spin-1/2 particle in the gravitational field of a massive body of radius $R$ which slightly exceeds the Schwarzschild radius $r_{s}$, possesses a dense spectrum of narrow resonances. Their lifetimes and density tend to infinity in the limit $R \rightarrow r_{s}$. We determine the cross section of the particle capture into these resonances and show that it is equal to the spin- $1 / 2$ absorption cross section for a Schwarzschild black hole. Thus black-hole properties may emerge in a non-singular static metric prior to the formation of a black hole.
\end{abstract}

PACS numbers: 04.62.+v, 04.70.Dy, 04.70.-s

\section{INTRODUCTION}

In this work we consider scattering of massless spin- $1 / 2$ particles by the gravitational field of finite-sized bodies whose radius $R$ slightly exceeds the Schwarzschild radius $r_{s}=2 G M / c^{2}$. Here $M$ is the mass of the body, $G$ is the universal gravitation constant, and $c$ is the speed of light. The spacetime around the body is described using a suitable metric to model its interior, which is joined at the surface of the body to the standard Schwarzschild metric outside. We find that for $R$ approaching $r_{s}$, the scattering is characterized by a dense spectrum of narrow resonances, i.e., metastable states whose lifetime and energy density tend to infinity in the black-hole limit. A particle that enters such states is trapped on the interior of the body for a time $\tau \sim \hbar / \Gamma_{n}$, where $\Gamma_{n}$ is the width of a given resonance.

For $R \rightarrow r_{s}$ both the energy spacing $D$ between the resonances and their width $\Gamma_{n}$ tend to zero, and the lifetime $\tau \rightarrow \infty$. At the same time the ratio $\Gamma_{n} / D$ for the fixed energy of the particle remains finite. This allows one to define the cross section for particle capture into these long-lived states using the optical model [1], i.e., averaging over a small energy interval containing many resonances. In doing so we recover the low-energy limit of the absorption cross section for a pure black hole (with the boundary condition of complete absorption at the event horizon) derived by Unruh: $\sigma=\frac{1}{4} \pi r_{s}^{2}$ for the $s_{1 / 2}$ and $p_{1 / 2}$ partial waves [2]. The total absorption cross section considering all partial-wave contributions is $\frac{1}{2} \pi r_{s}^{2}$, as only the $s_{1 / 2}$ and $p_{1 / 2}$ waves have nonvanishing cross sections at zero energy. Thus we observe that at low incident particle energies the absorption properties of a body with $R>r_{s}$ resemble those of a black hole.

It is worth noting that possible inelastic processes, such as radiation by the particles captured in the long-lived resonances, do not change $\sigma_{a}$. The presence of inelastic processes increases the total width of the resonances, $\Gamma_{\text {tot }}=\Gamma_{n}+\Gamma_{\text {inel }}$, but this quantity drops out of the energy averaged (optical) total capture cross section $\sigma_{a}$, leaving only the dependence on the elastic width $\Gamma_{n}[1]$.

As in the previous work on the scalar (spin-0) case [4], our calculations are performed twice: numerically (without approximations) and using analytical approximations, with good agreement between the two. In contrast to the spin-0 case we consider scattering for arbitrary angular momenta.

This work is closely related to the case of massive spin-1/2 particles trapped by a near-black-hole gravitational field, considered in [3]. The authors showed that the bound-state energy spectrum collapses and becomes quasi-continuous in the black-hole limit. The collapse of the positive-energy resonance spectrum in the black-hole limit found in this work shows similar behaviour.

The analytics presented are valid for interior metrics that satisfy certain physically reasonable constraints, detailed in later sections. In the present work we do not aim to consider real stars, rather we investigate the theoretical question of how quantum effects manifest themselves when a metric approaches the black hole metric. For numerical calculations we use two metrics which allow $r_{s} / R$ to be arbitrarily close to 1 without any metric singularities emerging. They are the Florides [5] and Soffel [6] metrics, which correspond to a system with vanishing radial stresses [7], and an modified Schwarzschild interior metric respectively.

\section{DIRAC EQUATION IN CURVED SPACETIME}

\section{A. Radial equation}

Consider a curved spacetime with the static, spherically symmetric metric:

$$
d s^{2}=a(r) d t^{2}-b(r) d r^{2}-r^{2} d \Omega^{2},
$$

where $a(r)$ and $b(r)$ are positive functions. The Dirac equation for a massless spin-1/2 particle in the above metric may be represented as two coupled equations (de- 
rived in [3]) for upper and lower components of the wavefunction $f(r)$ and $g(r)$, given by

$$
\begin{gathered}
\frac{d f(r)}{d r}+\sqrt{b(r)} \frac{\kappa}{r} f(r)-\varepsilon \sqrt{\frac{b(r)}{a(r)}} g(r)=0, \\
\frac{d g(r)}{d r}-\sqrt{b(r)} \frac{\kappa}{r} g(r)+\varepsilon \sqrt{\frac{b(r)}{a(r)}} f(r)=0 .
\end{gathered}
$$

Here $\kappa=\mp\left(j+\frac{1}{2}\right)$, where $j=l \pm \frac{1}{2}$ is the total angular momentum and $l$ is the orbital angular momentum. The equations (2) can be recast as the following second-order differential equation for $f(r)$ in a given partial wave

$$
\begin{aligned}
& f^{\prime \prime}(r)+\frac{b(r)}{2 a(r)}\left[\frac{a(r)}{b(r)}\right]^{\prime} f^{\prime}(r) \\
& +\left\{\frac{\varepsilon^{2} b(r)}{a(r)}+\frac{\kappa \sqrt{b(r)}}{r^{2}}\left[\frac{r a^{\prime}(r)}{2 a(r)}-\kappa \sqrt{b(r)}-1\right]\right\} f(r)=0 .
\end{aligned}
$$

\section{B. Interior solution}

Changing the radial variable to to the Regge-Wheeler "tortoise" coordinate $r^{*}$ defined by $d r^{*}=\sqrt{b(r) / a(r)} d r$, we can transform Eq. (3) to the following Schrödingerlike equation for $f\left(r^{*}\right)$,

$$
\frac{d^{2} f}{d r^{* 2}}+\left\{\varepsilon^{2}+\frac{\kappa r}{2 \sqrt{b(r)}}\left[\frac{a(r)}{r^{2}}\right]^{\prime}-\frac{\kappa^{2} a(r)}{r^{2}}\right\} f=0 .
$$

The metric outside of a massive nonrotating spherical body is given by the Schwarzschild solution,

$$
a(r)=1-r_{s} / r, \quad b(r)=\left(1-r_{s} / r\right)^{-1} .
$$

On the surface of the body $a(R)=1-r_{r} / R$. Since the metric is continuous, smooth and monotonic, then in the near-black-hole limit $\left(r_{s} \rightarrow R\right)$ the interior metric $a(r) \rightarrow 0$ for all $0 \leq r \leq R$, as the time slows down inside the gravitational potential. In this regime the first term in brackets in Eq. (4) dominates for all except very small energies, and for all distances, except near the origin. This means that the solution away from the origin describes free motion in the tortoise coordinate,

$$
f \simeq \sin \left(\varepsilon r^{*}+\phi\right)
$$

where the phase $\phi$ is determined by behaviour of the wave function near the origin.

In the vicinity of $r=0$, the dominant coefficient of $f\left(r^{*}\right)$ in Eq. (4) is given by the $r^{-2}$ centrifugal terms. For the specific metrics we consider (see Sec. IV), for $r \rightarrow 0, a(r) \simeq a(0)>0$ and $b(r) \simeq 1$. This also applies to a wider class of static solutions where the potential is harmonic near the origin. In this case, the centrifugal term in Eq. (4) is $\kappa(\kappa+1) / r^{* 2}=l(l+1) / r^{* 2}$, and the corresponding phase shift is given by $\phi=-l \pi / 2[1]$.
Hence, away from the origin the interior solution that is regular at the origin, is given by

$$
f(r)=\sin \left(\varepsilon \int_{0}^{r} \eta\left(r^{\prime}\right) d r^{\prime}-\frac{l \pi}{2}\right)
$$

where $\eta(r)=\sqrt{b(r) / a(r)}$. [Analysis of Eqs. (58) and (59) in [3] shows that for the regular solution, $f \propto r^{l+1}$ and $g \propto r^{l^{\prime}+1}$, which explains why the phase in Eq. (7) contains $l$ rather than $\kappa$.]

In what follows we solve the scattering problem by matching the logarithmic derivative of the exterior solution at $r=R$ to the logarithimic derivative of the interior solution, Eq. (7):

$$
\left.\frac{f^{\prime}(r)}{f(r)}\right|_{R}=\frac{\varepsilon R}{R-r_{s}} \cot \left(\varepsilon \Lambda(R)-\frac{l \pi}{2}\right),
$$

where

$$
\Lambda(R)=\int_{0}^{R} \eta(r) d r
$$

In Eq. (8) we also used the fact that

$$
\eta(R)=R /\left(R-r_{s}\right)
$$

since the interior metric matches Eq. (5) at $r=R$.

Note that in the black-hole limit $\left(R \rightarrow r_{s}\right)$ the function $\Lambda(R)$ tends to infinity. This means that for a fixed energy $\varepsilon$, the phase of the interior wave function (7) is large and the wave function oscillates rapidly, in close analogy with the case of massless scalar particles with $l=0$ [4].

\section{Exterior solution}

Using the exterior Schwarzschild metric, Eq. (5), in the radial wave equation (3), we obtain for $r>R$ :

$$
\begin{aligned}
& f^{\prime \prime}(r)+\left(\frac{1}{r-r_{s}}-\frac{1}{r}\right) f^{\prime}(r) \\
& +\left[\frac{\varepsilon^{2} r^{2}}{\left(r-r_{s}\right)^{2}}+\frac{\kappa\left(3 r_{s}-2 r\right)}{2 r^{3 / 2}\left(r-r_{s}\right)^{3 / 2}}-\frac{\kappa^{2}}{r\left(r-r_{s}\right)}\right] f(r)=0 .
\end{aligned}
$$

\section{Region $I(r \approx R)$}

For near-black-hole metrics $\left(R \approx r_{s}\right)$ we can keep only the most singular terms in the wave equation (11) near the boundary $(r \approx R)$, defined as Region I. Neglecting less singular terms and setting $r=r_{s}$ elsewhere, we have

$$
f^{\prime \prime}+\frac{1}{r-r_{s}} f^{\prime}+\left[\frac{\varepsilon^{2} r_{s}^{2}}{\left(r-r_{s}\right)^{2}}+\frac{\kappa}{2 r_{s}^{1 / 2}\left(r-r_{s}\right)^{3 / 2}}\right] f=0 .
$$

The exact solution of this equation are the Bessel functions $J_{4 i \varepsilon r_{s}}(\rho)$ and $Y_{4 i \varepsilon r_{s}}(\rho)$, where 
$\rho=\sqrt{8 \kappa} \sqrt[4]{\left(r-r_{s}\right) / r_{s}}$. At low energies $\varepsilon r_{s} \ll 1$, using the lowest-order terms in the expansion of the Bessel functions, gives the wave function in Region I as

$$
f_{1}(r)=\alpha_{1}+\beta_{1} \ln \left(\frac{r-r_{s}}{r_{s}}\right),
$$

where the constants $\alpha_{1}$ and $\beta_{1}$ are determined by matching with the interior solution at the boundary.

\section{Region $I I(r \gg R)$}

In this region the wave equation (11) takes the form of the nonrelativistic Schrödinger equation for a particle with momentum $\varepsilon$, angular momentum $l$ and unit mass in the attractive Coulomb field $Z / r$ with the charge $Z=$ $-\varepsilon^{2} r_{s}$. The exterior solution is thus a linear combination of the regular and irregular Coulomb functions,

$$
f_{2}(r)=\alpha_{2} F_{l}(\varepsilon r)+\beta_{2} G_{l}(\varepsilon r),
$$

which behave asymptotically as $F_{l} \sim \sin z$ and $G_{l} \sim$ $\cos z$, where $z=\varepsilon r+\varepsilon r_{s} \ln 2 \varepsilon r-l \pi / 2+\delta_{l}^{C}$ and $\delta_{l}^{C}=$ $\arg \left[\Gamma\left(l+1-i \varepsilon r_{s}\right)\right]$ is the Coulomb phase shift.

Following Unruh's matching procedure [2], we find the relationships between the coefficients in regions I and II, for $\kappa<0$ [10],

$$
\begin{aligned}
& \alpha_{2}=\frac{\alpha_{1}}{C_{l}(\varepsilon)}\left(\frac{4}{\varepsilon r_{s}}\right)^{|\kappa|}, \\
& \beta_{2}=-\frac{\beta_{1} C_{l}(\varepsilon)}{4}\left(\frac{\varepsilon r_{s}}{4}\right)^{|\kappa|-1}
\end{aligned}
$$

and for $\kappa>0$,

$$
\begin{aligned}
\alpha_{2} & =\frac{\beta_{1}}{4 C_{l}(\varepsilon)(2 \kappa+1)}\left(\frac{4}{\varepsilon r_{s}}\right)^{\kappa+1}, \\
\beta_{2} & =\alpha_{1} C_{l}(\varepsilon)(2 \kappa+1)\left(\frac{\varepsilon r_{s}}{4}\right)^{\kappa} .
\end{aligned}
$$

In these equations $C_{l}(\varepsilon)$ is the Coulomb factor,

$$
C_{l}(\varepsilon)=2^{l} e^{\pi \varepsilon r_{s} / 2} \frac{\left|\Gamma\left(l+1-i \varepsilon r_{s}\right)\right|}{(2 l+1) !} .
$$

For $\varepsilon r_{s} \ll 1$ this factor is a constant, $C_{l} \simeq 1 /(2 l+1)$ !!.

\section{S-MATRIX AND RESONANCES}

The solution to Eq. (11) at large distances can be written as

$$
f(r) \sim A e^{i z}+B e^{-i z},
$$

The ratio of the coefficients in front of the outgoing and incoming waves defines the $S$-matrix,

$$
S_{\kappa}=-\frac{A}{B} \exp \left(2 i \delta_{l}^{C}\right),
$$

and the short-range phase shift $\delta$, via $e^{2 i \delta} \equiv-A / B$. Comparing Eq. (13) with (19), we obtain

$$
A=\frac{\beta_{2}-i \alpha_{2}}{2}, \quad B=\frac{\beta_{2}+i \alpha_{2}}{2},
$$

which yields

$$
S_{\kappa}=-\frac{1-i \alpha_{2} / \beta_{2}}{1+i \alpha_{2} / \beta_{2}} \exp \left(2 i \delta_{l}^{C}\right) .
$$

Using the values of $\alpha_{2}$ and $\beta_{2}$ determined previously we have

$$
\begin{array}{ll}
\frac{\alpha_{2}}{\beta_{2}}=-\frac{4}{C_{l}^{2}}\left(\frac{4}{\varepsilon r_{s}}\right)^{2|\kappa|-1} \frac{\alpha_{1}}{\beta_{1}} & (\kappa<0), \\
\frac{\alpha_{2}}{\beta_{2}}=\frac{1}{4 C_{l}^{2}(2 \kappa+1)^{2}}\left(\frac{4}{\varepsilon r_{s}}\right)^{2 \kappa+1} \frac{\beta_{1}}{\alpha_{1}} & (\kappa>0) .
\end{array}
$$

The elastic scattering cross section is proportional to $\mid 1-$ $\left.S_{\kappa}\right|^{2}$. When the $S$-matrix varies rapidly as a function of energy, the cross section displays resonance maxima for $S_{\kappa} \approx-1$. At low energies, $\varepsilon r_{s} \ll 1$, the Coulomb phase shift is small (and it varies slowly with energy), and the resonances occur for $\alpha_{2} / \beta_{2}=0$ [see Eq. (22)]. This corresponds to

$$
\frac{\alpha_{1}}{\beta_{1}}=0 \quad(\kappa<0), \quad \frac{\beta_{1}}{\alpha_{1}}=0 \quad(\kappa>0) .
$$

These ratios are determined by matching the solution for the Schwarzschild exterior metric with the interior solution.

\section{A. Resonance energies}

Matching the logarithmic derivative of the exterior solution (12) at $r=R$ to that from Eq. (8) yields

$$
\frac{\alpha_{1}}{\beta_{1}}=\frac{\tan [\varepsilon \Lambda(R)-l \pi / 2]}{\varepsilon R}-\ln \left(\frac{R-r_{s}}{R}\right) .
$$

The resonance conditions, Eq. (25), translate into the tangent function tending to either zero or infinity depending on the sign of $\kappa$. (There is a small offset due to the logarithmic term but this is negligible in the $r_{s} \rightarrow R$ limit, since $\Lambda(R)$ increases much faster.) Hence, we find the expression for the resonance energies

$$
\varepsilon_{n}=\frac{\pi[n+(|\kappa|-1) / 2]}{\Lambda(R)},
$$

which is valid for both $\kappa<0$ and $\kappa>0$.

Since $\Lambda(R) \rightarrow \infty$ for $R \rightarrow r_{s}$, the energies of all resonances tend to zero in the black-hole limit, and the resonance spectrum "collapses", as its density tends to infinity. A similar collapse of spectrum is also seen in the bound state case detailed in [3]. 


\section{B. Resonance widths}

The full resonance condition states that resonances correspond to poles of the $S$-matrix at energies $\varepsilon=$ $\varepsilon_{n}-i \Gamma_{n} / 2$ which lie below the real axis in the complexenergy plane [1]. According to Eq. (22), this occurs when

$$
1+\frac{i \alpha_{2}}{\beta_{2}}=0
$$

To determine the resonance widths $\Gamma_{n}$, we use Eqs. (23) and (24) and expand the ratio $\alpha_{1} / \beta_{1}\left(\right.$ for $\kappa<0$ ) or $\beta_{1} / \alpha_{1}$ (for $\kappa>0$ ) to first order about the resonance energy $\varepsilon_{n}$. For example, for negative $\kappa$ we use

$$
\frac{\alpha_{1}}{\beta_{1}} \simeq\left(\frac{\alpha_{1}}{\beta_{1}}\right)^{\prime}\left(\varepsilon-\varepsilon_{n}\right)=\left(\frac{\alpha_{1}}{\beta_{1}}\right)^{\prime}\left(-i \Gamma_{n} / 2\right),
$$

thus Eqn. (28) may be written (for negative $\kappa$ ) as

$$
1+f(\varepsilon) \frac{\Gamma_{n}}{2}\left(\frac{\alpha_{1}}{\beta_{1}}\right)^{\prime}=0,
$$

where the prime denotes differentiation with respect to $\varepsilon$, the derivative is evaluated using Eq. (26) at the point where $\tan [\varepsilon \Lambda(R)-l \pi / 2]=0$, and $f(\varepsilon)$ is defined by Eqn. (23). Hence, we obtain for $\kappa<0$,

$$
\Gamma_{n}=\frac{2 C_{l}^{2} R}{\Lambda(R) r_{s}}\left(\frac{\varepsilon r_{s}}{4}\right)^{2|\kappa|},
$$

and following a similar procedure expanding $\beta_{1} / \alpha_{1}$ for $\kappa>0$,

$$
\Gamma_{n}=\frac{2 C_{l}^{2}(2 \kappa+1)^{2} r_{s}}{\Lambda(R) R}\left(\frac{\varepsilon r_{s}}{4}\right)^{2 \kappa} .
$$

The widths must be evaluated at $\varepsilon=\varepsilon_{n}$ from Eq. (27).

\section{Cross sections}

Comparing the above expressions for the widths with the resonance energy spacing $D=\varepsilon_{n+1}-\varepsilon_{n}=\pi / \Lambda(R)$ [see Eq. (27)], we see that $\Gamma_{n} \ll D$ at low energies, i.e., $\varepsilon r_{s} \ll 1$. In this case one can consider the cross section of capture into the resonances. This cross section corresponds to the optical-model energy-averaged absorption cross section [1], which is given by

$$
\sigma_{\kappa}^{(a)}=|\kappa| \frac{2 \pi^{2} \Gamma_{n}}{\varepsilon^{2} D}
$$

for a particular partial wave $\kappa$.

Using Eqs. (31) and (32), we obtain the resonant absorption cross sections for the near-black-hole metric:

$$
\sigma_{\kappa}^{(a)}= \begin{cases}\frac{1}{4} \pi r_{s}^{2}|\kappa| C_{l}^{2}\left(\frac{\varepsilon r_{s}}{4}\right)^{2|\kappa|-2} & (\kappa<0), \\ \frac{1}{4} \pi r_{s}^{2}|\kappa| C_{l}^{2}(2 \kappa+1)^{2}\left(\frac{\varepsilon r_{s}}{4}\right)^{2 \kappa-2} & (\kappa>0) .\end{cases}
$$

Note that, unlike the resonance energies and widths, the resonance capture cross sections do not contain $\Lambda(R)$, and hence, are independent of the interior metric used, as long as the metric satisfies the assumptions made in Sec. (II B).

In the zero-energy limit only two partial waves give nonzero contributions, namely, $s_{1 / 2}$ and $p_{1 / 2}(|\kappa|=1)$. The corresponding cross sections $\sigma_{\mp 1}^{(a)}=\frac{1}{4} \pi r_{s}^{2}$ are in agreement with Unruh's result $\sigma_{\text {tot }}^{(a)}=\sum_{\kappa} \sigma_{\kappa}^{(a)}=\frac{1}{2} \pi r_{s}^{2}$ $[2]$.

\section{NUMERICAL RESULTS FOR SPECIFIC INTERIOR METRICS}

In this section we present calculations involving two specific interior metrics that allow the $r_{s} \rightarrow R$ limit to be taken: the Florides [5] and Soffel [6] metrics. Specifically, we verify the analytics provided previously with numerically calculated resonance widths and energies via the short range phase shift. To calculate this short range phase $\delta_{\mathrm{I}}$ we solve the second-order differential equation (3) numerically, for given $a(r)$ and $b(r)$, with the boundary condition $f(0)=r^{l+1}, f^{\prime}(0)=(l+1) r^{l}$ using Mathematica [9]. This solution provides a real boundary condition for the exterior wave function at $r=R$. (We set $R=1$ in the numerical calculations). Equation (11) is then integrated outwards to large distances $r \gg r_{s}$. In this region Eq. (11) takes the form of a nonrelativistic Shrödinger equation for a particle with momentum $\varepsilon$ and unit mass in the Coulomb potential with charge $Z=-r_{s} \varepsilon^{2}$. Hence, we match the solution with the asymptotic form [1]

$$
f(r) \propto \sin \left[\varepsilon r-(Z / \varepsilon) \ln 2 \varepsilon r+\delta_{C}+\delta-l \pi / 2\right]
$$

where $\delta_{C}=\arg \Gamma(1+l+i Z / \varepsilon)$ is the Coulomb phase shift, and determine the short-range phase shift $\delta$.

The numeric widths and positions of the resonances are then extracted from this phase shift by fitting it to the Breit-Wigner profile

$$
\delta(\varepsilon)=A+\arctan \left[\frac{\varepsilon-\varepsilon_{n}}{\Gamma_{n} / 2}\right]
$$

in the region of an isolated resonance $\left(\varepsilon \approx \varepsilon_{n}\right)$, where $A$ is a constant offset.

\section{A. Florides Interior}

The Florides metric is characterized by

$$
a(r)=\frac{\left(1-r_{s} / R\right)^{3 / 2}}{\sqrt{1-r_{s} r^{2} / R^{3}}}, \quad b(r)=\left(1-\frac{r_{s} r^{2}}{R^{3}}\right)^{-1} .
$$




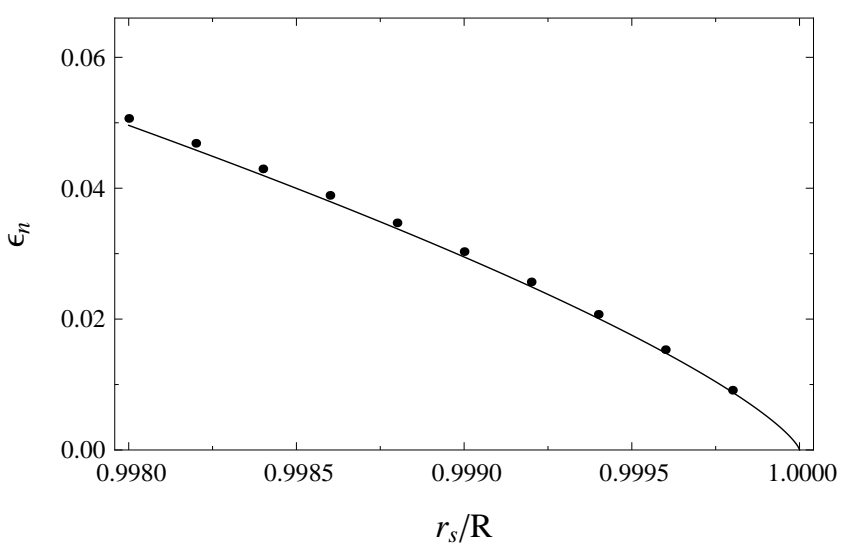

FIG. 1. Energies of the $n=2$ resonance in the Florides metric. Closed circles indicate numeric data, the solid line indicates analytic $\varepsilon_{n}$ given by Eqn. (27) with $\lambda$ given by Eqn. (38).

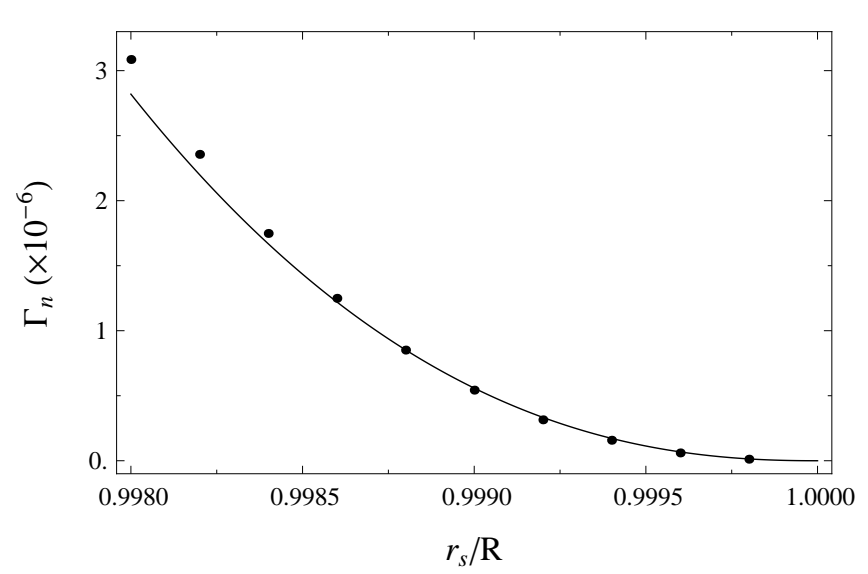

FIG. 2. Width of the $n=2$ resonance in the Florides metric. Closed circles indicate numeric data, the solid line indicates analytic $\Gamma_{n}$ given by Eqn. (31) with $\lambda$ given by Eqn. (38).

This in turn leads to

$$
\begin{aligned}
\Lambda(R)_{\mathrm{F}} \stackrel{r_{s} \rightarrow R}{=} \frac{\pi^{3 / 2} R}{\sqrt{2} \Gamma(1 / 4) \Gamma(5 / 4)\left(1-r_{s} / R\right)^{3 / 4}} \\
\quad \approx 1.198\left(R-r_{s}\right)^{-3 / 4} .
\end{aligned}
$$

The resulting resonance energies and widths are compared with their numeric counterparts in Figures (1) and (2) respectively.

\section{B. Soffel Interior}

The Soffel metric is characterized by [6]

$$
a(r)=\left(1-\frac{r_{s}}{R}\right) \exp \left[-\frac{r_{s}\left(1-r^{2} / R^{2}\right)}{2 R\left(1-r_{s} / R\right)}\right],
$$

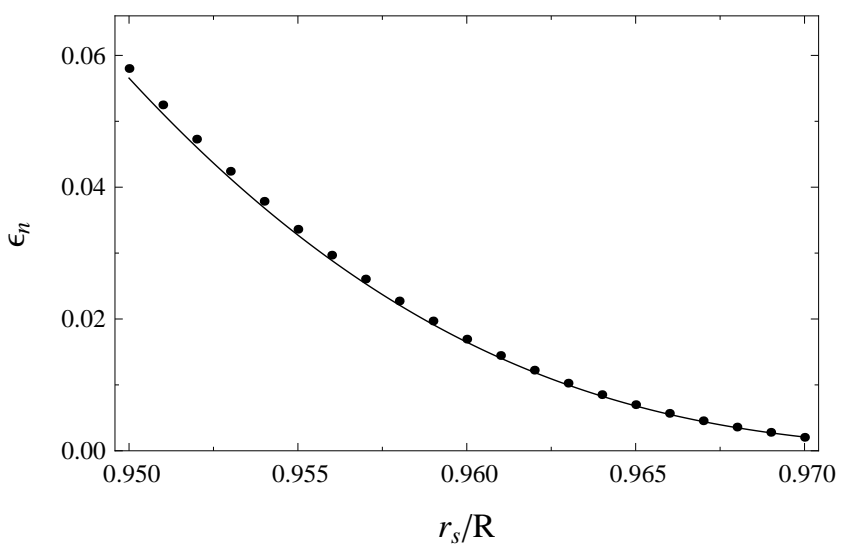

FIG. 3. Energies of the $n=4$ resonance in the Soffel metric. Closed circles indicate numeric data, the solid line indicates analytic $\varepsilon_{n}$ given by Eqn. (27) with $\lambda$ given by Eqn. (40).

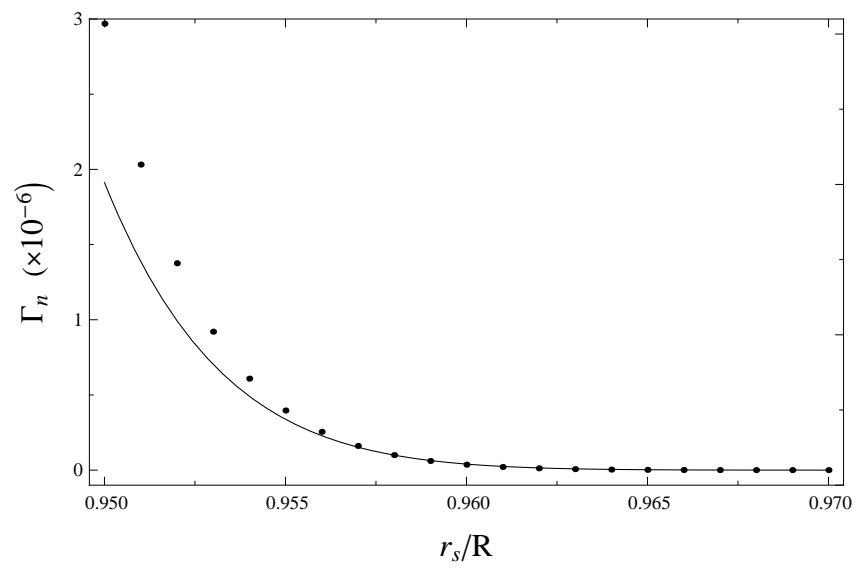

FIG. 4. Widths of the $n=4$ resonance in the Soffel metric. Closed circles indicate numeric data, the solid line indicates analytic $\Gamma_{n}$ given by Eqn. (31), with $\lambda$ given by Eqn. (40).

with $b(r)$ equal to that of the Florides case. This in turn leads to

$$
\Lambda(R)_{\mathrm{So}} \stackrel{r_{s} \rightarrow R}{=} R \sqrt{\pi} \exp \left[\frac{r_{s} / R}{4\left(1-r_{s} / R\right)}\right]
$$

Analytic and numeric $\varepsilon_{n}$ and $\Gamma_{n}$ for the Soffel metric are compared in Figures (3) and (4) respectively.

\section{CONCLUSIONS}

The problem of scattering of low-energy spin-1/2 particles from a massive static spherical body has been considered. We have shown that as in the spin-0 case, approaching the black hole case gives rise to a dense spectrum of long lived resonances. Similar to the scalar case, 
we show that the existence and structure of these resonances gives rise to effective absorption in the purely potential scattering problem. This allows us to construct an absorption cross section for bodies near the black hole threshold which matches known results for the pure black hole case in the low energy limit.

\section{ACKNOWLEDGMENTS}

We thank A. V. Korol for useful discussions.
[1] L. D. Landau and E. M. Lifshitz, Quantum Mechanics, 3rd ed. (Butterworth-Heinemann, Oxford, 1977).

[2] W. G. Unruh, Phys. Rev. D 14, 3251 (1976); Thesis, Princeton Univ., 1971 (unpublished).

[3] A. F. Spencer-Smith, G. H. Gossel, J. C. Berengut, and V. V. Flambaum, Gen. Rel. Gravit., 45, 613 (2013).

[4] V. V. Flambaum, G. H. Gossel, and G. F. Gribakin, Phys. Rev. D 85, 084027 (2012).

[5] P. S. Florides, Proc. R. Soc. Lond. A 337, 529 (1974).

[6] M. Soffel, B. Müller, and W. Greiner, J. Phys. A 10, 551 (1977).

[7] For $r_{s}<2 R / 3$ the Florides metric may represent the gravitational field inside a cluster of particles moving in randomly oriented circular orbits for larger $R$ (Einstein cluster). In the case where $r_{s}>2 R / 3$ the Florides met- ric does not correspond to any macroscopic object, but nevertheless represents a valid solution to the field equations. The 'physical reasonableness' of such a solution is discussed in both N. K. Kofinti, Gen. Relativ. Gravit. 17, 245 (1985) and L. Herrera and N. O. Santos, Phys. Rep. 286, 53 (1997).

[8] M. Yu. Kuchiev and V. V. Flambaum, Phys. Rev. D 70, 044022 (2004).

[9] Mathematica, Version 7.0 (Wolfram Research, Inc., Champaign, IL, 2008).

[10] Note that equation (46) in Unruh's paper [2] contains a misprint: the sign of the second term should be minus, not plus. Consequently, the right-hand side of the second equation (51) should also contain a minus. 